\title{
Correction to: A Randomized Controlled Trial of a Mini Low- Carbohydrate Diet and an Energy-Controlled Diet Among Japanese Patients With Type 2 Diabetes
}

\author{
Masayo Kimura a, b, Yoshinobu Kondo ${ }^{\text {a }}$, Kazutaka Aokia, c , Jun Shirakawa ${ }^{a}$, Hiroshi Kamiyama a, b, \\ Kazunari Kamiko, ${ }^{a}$, Shigeru Nakajima ${ }^{\text {, }}$ Yasuo Terauchi ${ }^{a}$,
}

This is to correct the published article, Journal of Clinical Medicine Research, 2018;10(3):182-188. DOI:10.14740/jocmr3281w.

Due to the lack of some values in the previous version, Tables 1 and 2 and Figures 3 and 4 are slightly changed. The corrected tables and figures were described below.

In page 185, right paragraph, "However, there was a difference in LDL-cholesterol levels between the two groups $(\mathrm{P}=$ 0.018)." should be deleted, and Results for Figure 3 and Figure 4 should read "No difference was seen in the HbAlc of patients in either group at week 12, as compared with the baseline (mini-LCD: $\mathrm{P}=0.40$; ECD: $\mathrm{P}=0.10$ ) (Fig. 3). Patients in the mini-LCD group showed a significant difference in body weight at week 12 , as compared with the baseline $(\mathrm{P}=0.03)$; however, no significant difference was seen in patients in the ECD group $(\mathrm{P}=0.50)$ (Fig. 4)".

In page 187, left paragraph, the results about protein intake should read "There was a tendency to show the difference in protein intake between the two groups from the baseline and week 12 (mini-LCD: $3.7 \pm 4.6$; ECD: $-1.9 \pm 3.5$; $\mathrm{P}=0.34$ )".

In page 187, right paragraph, the Discussion about propensity score matching should read "A difference in HbAlc between the two groups or from week 0 to 12 after propensity score matching showed no significant changes.", and the following description should be deleted: "However, an increase in protein intake was observed in the mini-LCD group from the baseline to week $12(\mathrm{P}=0.045)$ " should be deleted.

In page 188, left paragraph, the Discussion about body weight changes should read "Previous reports have demonstrated that low carbohydrate diets are associated with weight loss in obese patients $[4,13,14]$. The present study also demonstrated that the mini-LCD diet tended to decrease the body weight."

The authors declare that these minor changes do not affect the conclusion of this study solely.

${ }^{a}$ Department of Endocrinology and Metabolism, Graduate School of Medicine, Yokohama City University, Yokohama 236-0004, Japan

${ }^{b}$ Nakajima Naika Clinic, Yokosuka 238-0011, Japan

'Internal Medicine, Kanagawa Dental University, Yokosuka, Japan

${ }^{\mathrm{d} C}$ Corresponding Author: Yasuo Terauchi, Department of Endocrinology and Metabolism, Graduate School of Medicine, Yokohama City University, Fukuura 3-9, Kanazawa-ku, Yokohama 236-0004, Japan.

Email: terauchi-tky@umin.ac.jp 
Table 1. Baseline Characteristics Before and After Propensity Score Matching

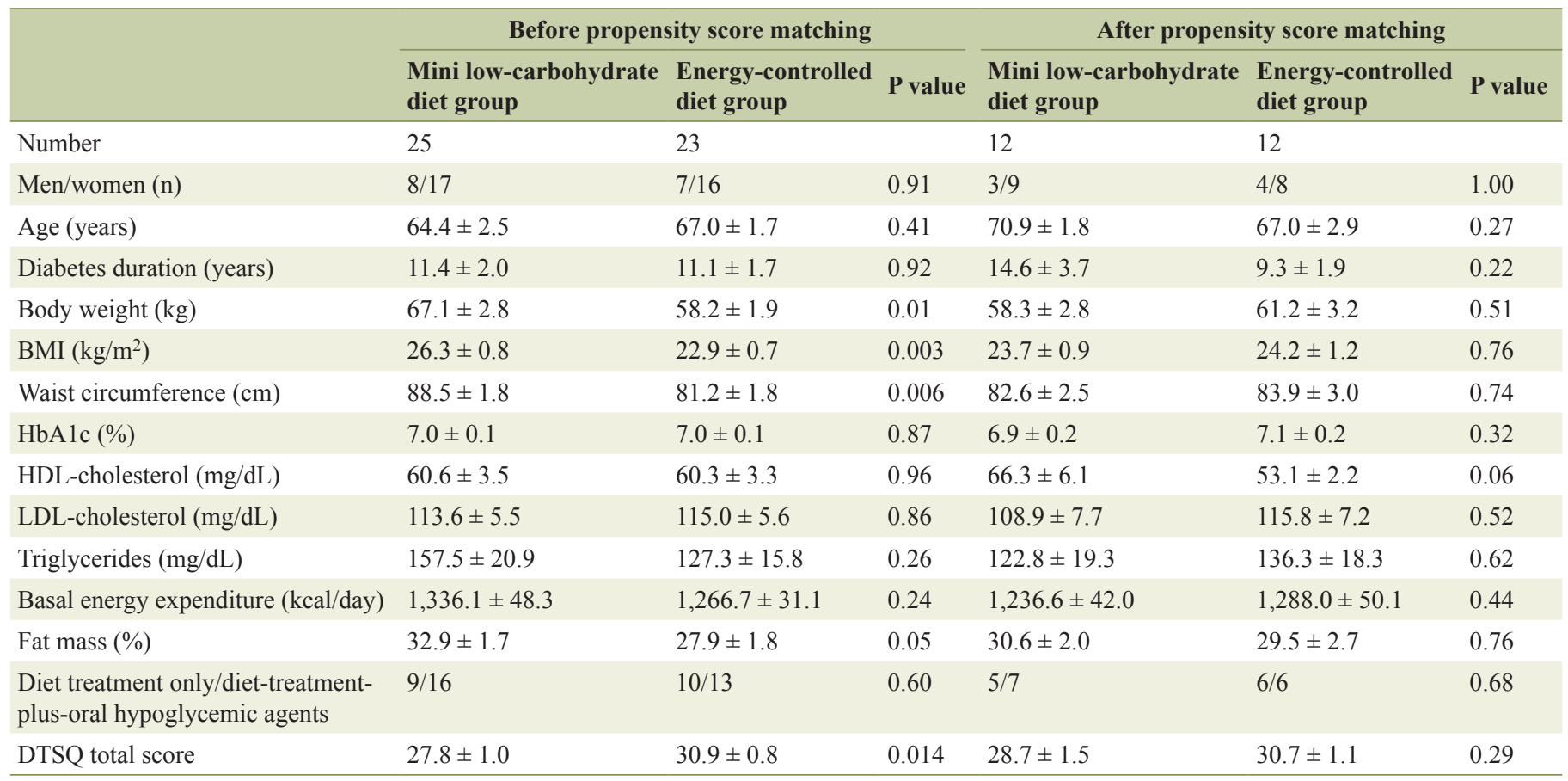

Data are expressed as means \pm standard error. P values between two groups were determined using the $t$-test. One-to-one nearest-neighbor matching with replacement based on propensity score was performed. To test the approach and maximize the number of matching, the process was repeated without a replacement option, using calipers and logit of the propensity score. Calipers of width equal to 0.25 of the standard deviation of the logit score. BMI: body mass index; HbA1c: glycosylated hemoglobin; HDL: high-density lipoprotein; LDL: low-density lipoprotein. 


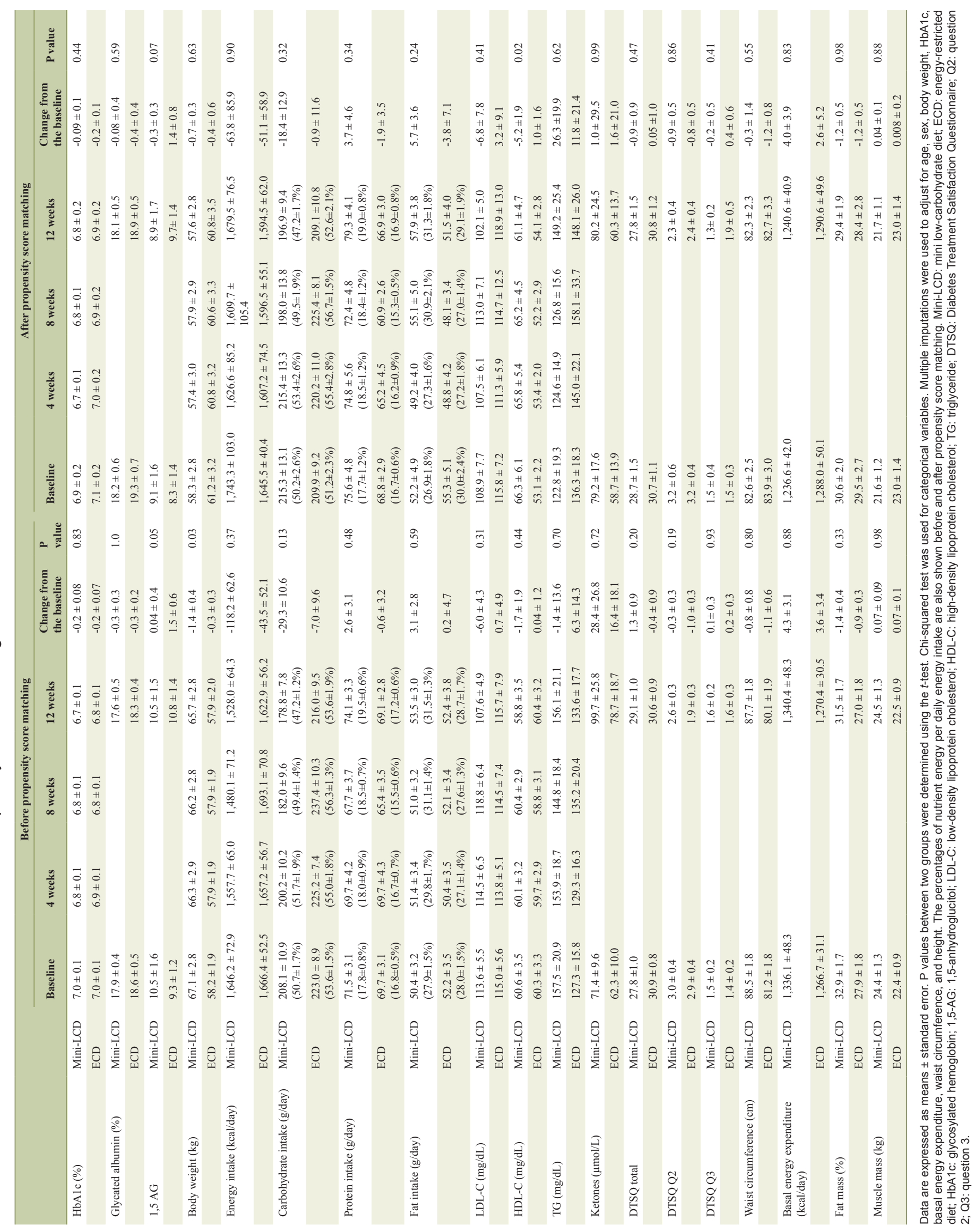




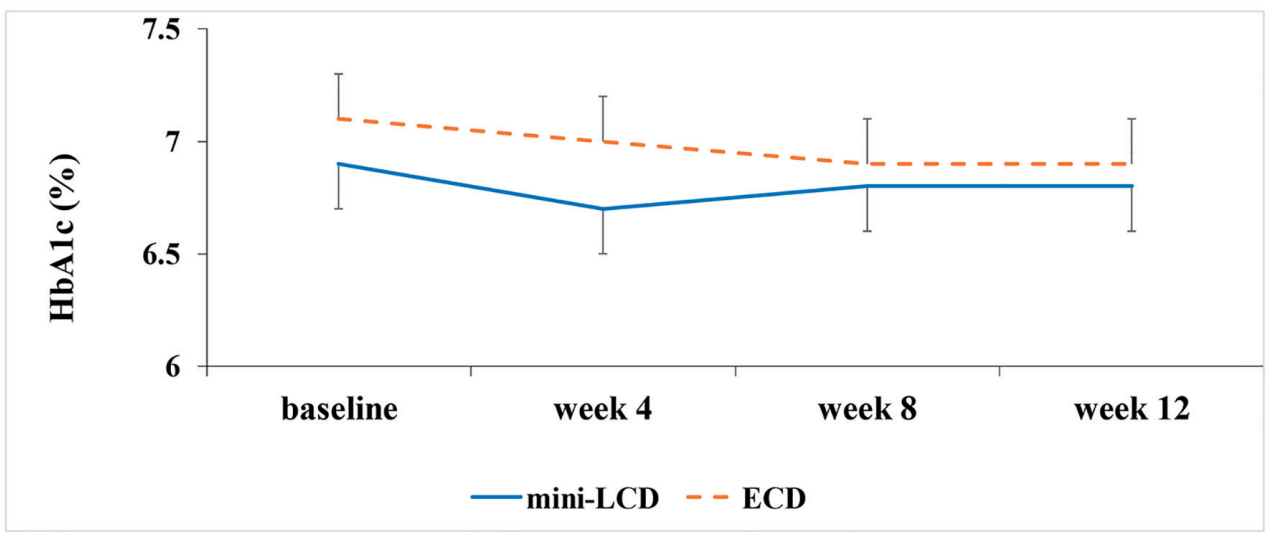

Figure 3. Change in HbA1c (\%). Mini-LCD: mini low-carbohydrate diet; ECD: energy-controlled diet; HbA1c: glycosylated hemoglobin.

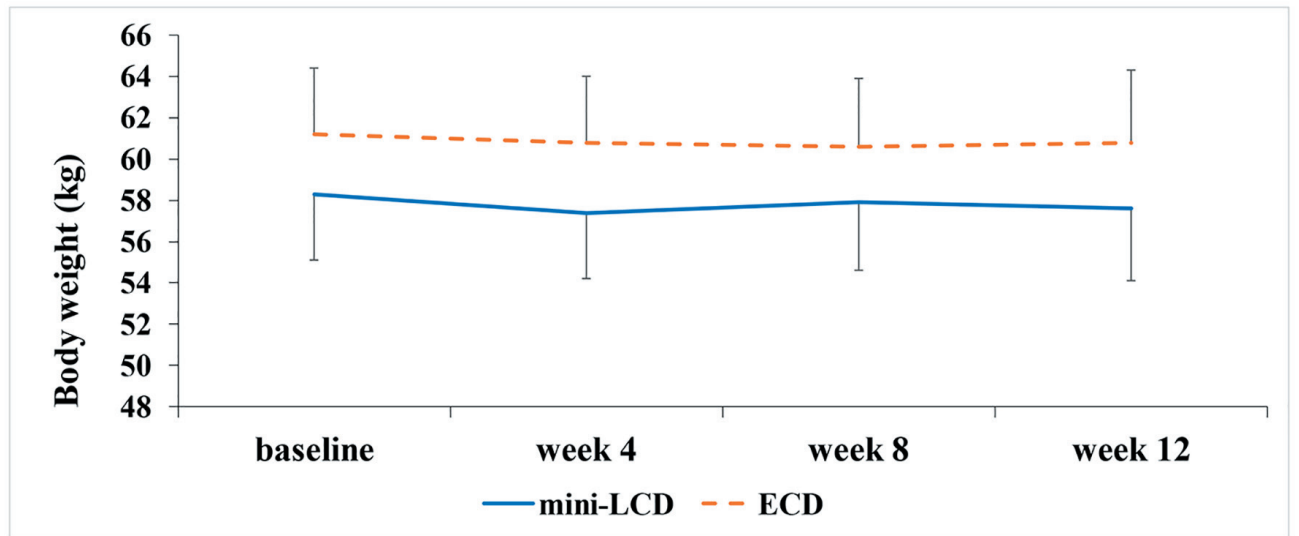

Figure 4. Change in body weight (kg). Mini-LCD: mini low-carbohydrate diet; ECD: energy-controlled diet. 